\title{
Review of key technologies and applications of intelligent transportation
}

\author{
Zhao Yan ${ }^{1, a}$, Cao Desheng ${ }^{2, b}$ and Ren Huazhong ${ }^{3, c}$ \\ ${ }^{1}$ China Transport Telecommunications \& Information Center, Beijing, China, \\ ${ }^{2}$ China Transport Telecommunications \& Information Center, Beijing, China \\ ${ }^{3}$ Peking University China \\ azhaoyan_susan@163.com, ${ }^{\mathrm{b}}$ caodesheng@cttic.cn, ${ }^{\mathrm{C}}$ renhuazhong@pku.edu.cn
}

Keywords: smart city, intelligent transportation, wireless sensor network, data mining, intelligent transportation cloud.

Abstract. As an important part of intelligent city construction, intelligent transportation has gained more and more attention. The concept and general structure of intelligent transportation are briefly introduced. The key technologies of wireless sensor network, data mining and intelligent transportation cloud are applied in intelligent transportation. Finally, the practical application of intelligent transportation is explained.

\section{Introduction}

All manuscripts must be in English, also the table and figure texts, otherwise we cannot publish your paper.

With the large-scale expansion of cities, the reform of infrastructure construction and management is relatively lagging behind. Therefore, the symptom benefits of urban diseases is serious. Traffic jams and traffic accidents are the most typical symptoms. In order to ease traffic congestion in implementation of the tail number limit line, However, it cannot fundamentally solve the problem. residents travel satisfaction will be more and more low, and the traffic congestion is becoming more and more serious, which is not conducive to the healthy development of the city. Therefore, the research of intelligent transportation is imperative. Intelligent transportation is a comprehensive interdisciplinary subject. If we want to really achieve the coordination of vehicle routing and comprehensive standards of urban transportation, we need more collaborative research. In order to make the researchers in the field of intelligent transportation more unified to understand the research situation. In this paper, the concept and frame of intelligent transportation are described in detail, and the key technologies and some specific applications of intelligent traffic are introduced in detail.

\section{Overview of intelligent traffic}

Using networking, space perception, cloud computing, mobile Internet and other new generation of information technology, intelligent transportation integrates traffic science, system method, artificial intelligence, knowledge mining theory and tools, with a comprehensive perception, the depth of integration, active service, scientific decision-making as the goal, through the dynamic construction of information service system in real time. The depth of mining transportation related data, forming problem analysis model, implementation ability, public decision-making ability, management ability, ability of public service industry to optimize the allocation of resources industry promotion, promote the operation and development of transportation safer, more efficient, more convenient, more economical, more environmentally friendly, more comfortable, driven by the transportation industry transformation upgrade. 


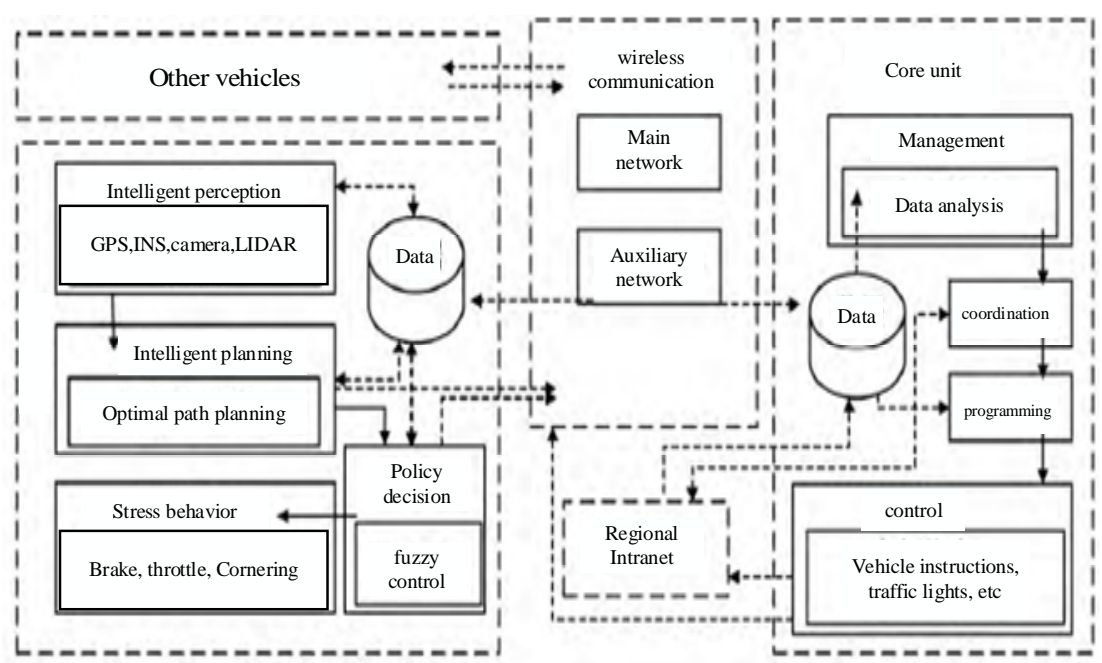

Fig. 1 The framework of Intelligent Transportation

\section{Key technologies of Intelligent Transportation}

\section{Identification and perception of the elements of traffic}

Intelligent recognition and wireless sensing technology is the most important technical means for identifying and perceiving objects, which is the foundation of the construction of the whole intelligent traffic. Wireless sensor network (Wire-less Sensor Networks, WSNs) is formed by the deployment of a large number of low-cost micro sensor nodes in the target in the monitoring area of the multi hop self-organizing network, nodes exchange information through wireless means, intelligent transportation network is flexible, low cost and easy to deploy in the sensor is divided into data acquisition node and the sink node.

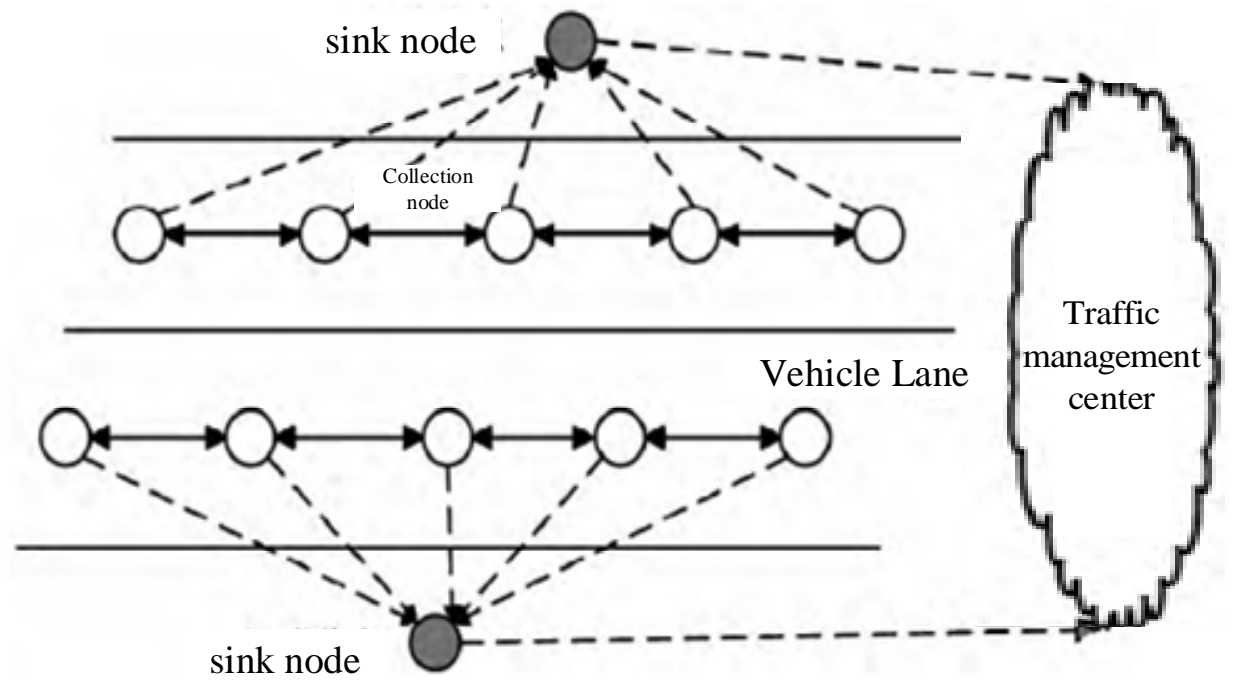

Fig. 2 Schematic diagram of road traffic management system

\section{Intelligent traffic cloud}

Intelligent transport cloud is a combination of intelligent traffic management technology of cloud computing mainly for transportation service industry, which makes full use of the mass storage, information security, unified resource processing and other advantages of cloud computing, sharing traffic field data and effective management provides a new way of thinking, which is divided into infrastructure as a Service(IaaS), platform as a service (PaaS), software as a service (SaaS) where IaaS is provided by the virtual server to use the PaaS Web service that can be provided directly and can be used directly in software application development or API development platform for the customers. 


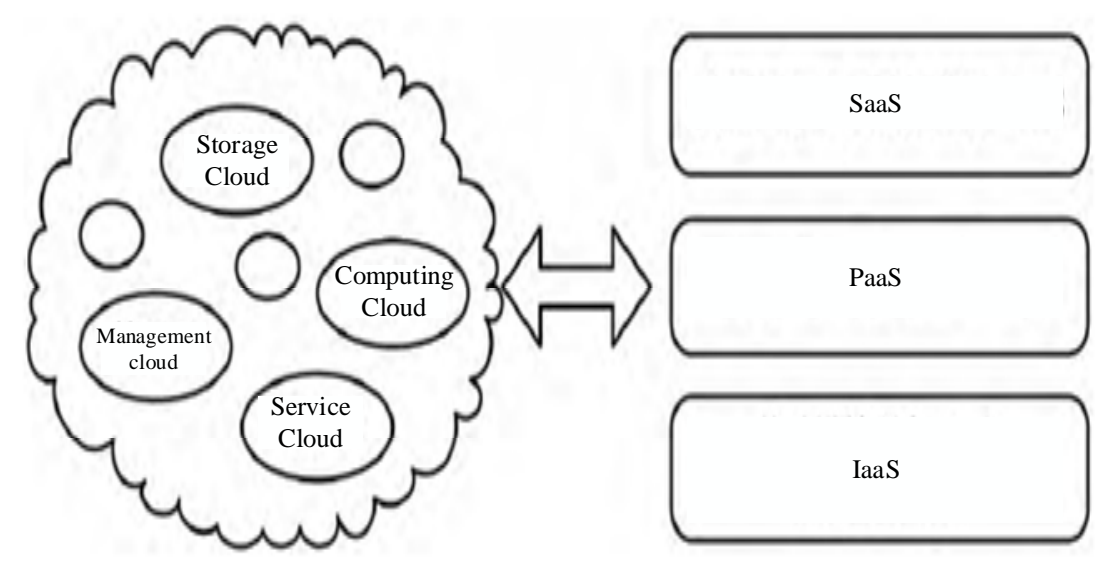

\section{Data processing technology}

Fig.3 Intelligent traffic cloud service level

The mass, diversity and isomerism of data in intelligent transportation all determine the complexity of the processing, simple to traffic facilities and the collection of traffic data. Simply, the collection of traffic facilities and traffic data and the determination and detection of traffic accidents need to process data in real time and accurately. The common data processing technology in intelligent transportation data fusion, data mining and data visualization, in addition, must also be selective to upload data, ensure the safety of personal data privacy.

\section{Integrated technology of intelligent transportation system}

The research on the integrated system technology is imminent. The system integration in the field of intelligent transportation can be divided into data integration and equipment integration. The data integration application way has two kinds, one is the internal data fusion single platform system, such as the fusion of multiple sensor information in the vehicle monitoring module, another is the analysis of multi-platform multi sensor data in different periods, the potential data to predict traffic information through fusion. Equipment integration is the current system needs to be smooth transition to the stage of intelligent transportation and cannot be replaced immediately. Therefore, we can formulate a unified intelligent transportation standard system and standardized management, the establishment of a standardized management platform, the intelligent transportation industry chain of government resources, enterprise resources, research resources together, then led by large enterprises to promote the coordinated intelligent transportation industry, eventually forming a complete system of traffic management.

\section{Development history of Intelligent Transportation}

Currently, the US, Japan and other countries have successfully applied the Internet of things to the construction of intelligent transportation. The research of road network collaboration and auxiliary driving function has also been put into trial operation and try to popularize smart terminals and transform the existing facilities. China started late, so far, the technology of car networking is not yet mature, but with the east wind of the construction of smart cities, intelligent transportation is also very fruitful.

\section{Conclusions}

In view of the fact that the importance of intelligent transportation in the smart city construction, should make full use of the advanced technology in the intelligent transportation technology, especially the popular cloud computing, data mining and system integration, constantly running and practical application environment, explore a new road traffic is more suitable for China's national conditions, for the levels of traffic and service objects to provide a more comprehensive and efficient traffic information service, so as to further promote the construction of smart city. 


\section{Acknowledgements}

This work was supported by key special project of National Key Research and Development Program (2017YFC0803900).

\section{References}

[1] C. M. Mertes, A. Schneider, D. Sulla-Menashe, A. Tatem, and B. Tan, "Detecting change in urban areas at continental scales with MODIS data," Remote Sens. Environ. 2015. 158. 331-347.

[2] J. Xia, M. Dalla Mura, J. Chanussot, P. Du, and X. He, "Random subspace ensembles for hyperspectral image classification with extended morphological attribute profiles," IEEE Trans. Geosci. Remote Sens., 2015. 53(9):4768-4786.

[3] K. S. Willis, "Remote sensing change detection for ecological monitoring in United States protected areas," Biol. Conserv. 2015. 182. 233-242.

[4] M. A. Bencherif, Y. Bazi, A. Guessoum, N. Alajlan, F. Melgani, and H. AlHichri, "Fusion of extreme learning machine and graph-based optimization methods for active classification of remote sensing images," IEEE Geosci. Remote Sensing Letters.2015. 12(3)L:527-531.

[5] P. Zhong and R. Wang, Jointly learning the hybrid CRF and MLR model for simultaneous denoising and classification of hyperspectral imagery, IEEE Trans. Neural Netw. Learn.2014,1319-1334.

[6] M. Hussain, D. Chen, A. Cheng, H. Wei, and D. Stanley, Change detection from remotely sensed images: From pixel-based to object-based aroaches, ISPRS J. Photogramm. Remote Sensig. 2013.80. 91-106.

[7] M. Turker, B. T. San. 2004. Detection of collapsed buildings caused by the 1999 Izmit Turkey earthquake through digital analysis of post-event aerial photographs [J]. INT.J. Remote Sensing, 25(21):4701-4714.

[8] Wang X Q, Dou A X, Sun G Q.2013. Intensity Assessment of the 2010 Yushu MS7. 1 Earthquake Based on Synthetic Seismic Damage Index[J]. Earthquake. 33(2):1-10. 